\title{
The influence of beam model differences in the comparison of dose calculation algorithms for lung cancer treatment planning
}

\author{
Indrin J Chetty, Mihaela Rosu, Daniel L McShan, Benedick A Fraass \\ and Randall $K$ Ten Haken
}

Department of Radiation Oncology, The University of Michigan, Ann Arbor, MI 48109-0010, USA

E-mail: indrin@med.umich.edu

Received 1 April 2004, in final form 23 September 2004

Published 17 February 2005

Online at stacks.iop.org/PMB/50/801

\begin{abstract}
In this study, we show that beam model differences play an important role in the comparison of dose calculated with various algorithms for lung cancer treatment planning. These differences may impact the accurate correlation of dose with clinical outcome. To accomplish this, we modified the beam model penumbral parameters in an equivalent path length (EPL) algorithm and subsequently compared the EPL doses with those generated with Monte Carlo (MC). A single AP beam was used for beam fitting. Two different beam models were generated for EPL calculations: (1) initial beam model (init_fit) and (2) optimized beam model (best_fit), with parameters optimized to produce the best agreement with MC calculated profiles at several depths in a water phantom. For the $6 \mathrm{MV}$, AP beam, EPL(init_fit) calculations were on average within $2 \% / 2 \mathrm{~mm}(1.4 \mathrm{~mm}$ max.) agreement with $\mathrm{MC}$; the agreement for EPL(best_fit) was $2 \% / 0.5 \mathrm{~mm}$ (1.0 mm max.). For the $15 \mathrm{MV}$, AP beam, average agreements with $\mathrm{MC}$ were $5 \% / 2 \mathrm{~mm}(7.4 \% / 2.6 \mathrm{~mm}$ max.) for EPL(init_fit) and 2\%/1.0 mm (1.3 mm max.) for EPL(best_fit). Treatment planning was performed using a realistic lung phantom using 6 and $15 \mathrm{MV}$ photons. In all homogeneous phantom plans, EPL(best_fit) calculations were in better agreement with MC. In the heterogeneous $6 \mathrm{MV}$ plan, differences

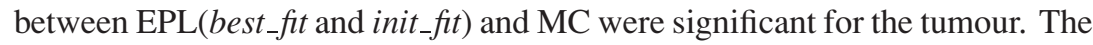
EPL(init_fit), unlike the EPL(best_fit) calculation, showed large differences in the lung relative to MC. For the $15 \mathrm{MV}$ heterogeneous plan, clinically important

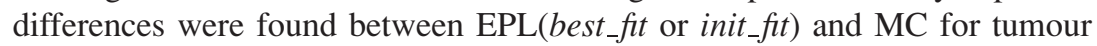
and lung, suggesting that the algorithmic difference in inhomogeneous tissues was most influential in this case. Finally, an example is presented for a $6 \mathrm{MV}$ conformal clinical treatment plan. In both homogeneous and heterogeneous cases, differences between EPL(best_fit) and MC for lung tissues were smaller

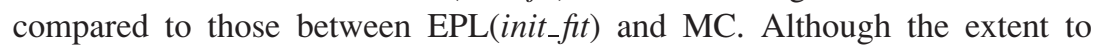


which beam model differences impact the dose comparisons will be dependent upon beam parameters (orientation, field size and energy), and the size and location of the tumour, this study shows that failing to correctly account for beam model differences will lead to biased comparisons between dose algorithms. This may ultimately hinder our ability to accurately correlate dose with clinical outcome.

(Some figures in this article are in colour only in the electronic version)

\section{Introduction}

Conventional dose calculation algorithms are typically based on parameterizations of dose measurements in a water phantom. These methods usually employ analytical models with various parameters that can be adjusted to 'optimize' the agreement with depth dose and profile measurements over a range of field sizes. However, in clinical treatment planning, and particularly in regions with inhomogeneous tissues, these algorithms-which employ simplistic radiological path length type corrections for inhomogeneities-have been shown by Arnfield et al (2000) (among many others) to be inaccurate. With physics-based methods, such as Monte Carlo (MC), beam models may be developed from explicit particle simulation of the treatment head (phase space), or by characterization of information from the phase space, and in some cases may be empirically based (Rogers et al 1995, DeMarco et al 1998, Deng et al 2000, Schach von Wittenau et al 2000, Fix et al 2001, Heath and Seuntjens 2003). Measurements are used mainly for the purposes of verification of the beam models. An important consideration in the comparison of MC with conventional methods is to be able to distinguish issues related to differences in the beam model (i.e., in the characterization of radiation from the treatment head) versus those due to calculational differences in the heterogeneous patient tissues (i.e., algorithmic differences due purely to the inhomogeneity correction methods). Although in practice it is difficult to separate these two issues, testing can be designed to emphasize beam model versus algorithmic differences and vice versa. For example, beam model differences may be best elucidated using larger field sizes in water phantoms, where differences in the energy spectra caused by increased scattering from structures, such as the flattening filter, are accentuated. On the other hand, algorithmic differences may be better tested using small field sizes, higher energy beams, in heterogeneous media, where lateral electronic disequilibrium becomes important.

In this work, we show that beam model differences (found when comparing algorithms in homogeneous media) may substantially influence the algorithmic comparison in the heterogeneous setting. These differences are especially important in the consideration of dose/volume/response correlations; if the beam models are not 'matched' in the homogeneous case, then the doses may be significantly different in low-density patient tissues, resulting in inaccurate estimation of the dose to such normal structures. The quantification of the differences resulting from beam model effects versus those from inhomogeneity effects is therefore essential part of performing an unbiased comparison of the algorithmic differences in the inhomogeneous setting. This type of analysis will place the various inhomogeneity correction methods on an equal footing to ultimately perform accurate correlations of the different dose distributions with lung cancer patient outcome (local failure and normal tissue complications, e.g., radiation pneumonitis). 


\section{Methods and materials}

\subsection{Dose algorithms}

The MC dose algorithm used in this study is the dose planning method (DPM) originally developed by Sempau et al (2000). DPM has been optimized for coupled photon/electron beam radiotherapy class calculations - the transport package employs several features for calculations as such. Some of these transport schemes include (a) the use of a step size independent multiple scattering theory, (b) the use of a 'random hinge' scheme in transporting charged particles from point to point in the medium, (c) the use of large electron steps which affords the ability to traverse many voxels before sampling a multiple scattering angle and (d) the use of Woodcock tracking to reduce the overheads associated with transporting photons across boundaries. Other details of the electron/photon transport model used in DPM along with electron beam accuracy and efficiency comparisons are provided in the paper by Sempau et al (2000). DPM has been integrated within our in-house treatment planning system (UMPlan) using a virtual source model to characterize the radiation phase space, and is currently being used for a variety of treatment planning applications (Chetty et al 2003, 2004). The MC virtual source model used for patient-specific treatment planning is similar to that previously developed by Chetty et al (2000), and is described at length in that paper. A brief description of the source model is provided here. The treatment head components of a Varian 21EX linear accelerator (Varian Associates, Palo Alto, CA) were simulated using the BEAMnrc MC code (CNRC, Ottawa, ON). A virtual source model was reconstructed from the phase space distribution to sample the source particle's position, energy. Direction is determined from the particle's sampled starting position assuming the particle emerged from a point. Arbitrary field shapes are simulated by multiplying the uncollimated fluence map by a matrix describing the multi-leaf collimator (MLC) configuration. In order to account for the finite width of the target and leaf edge penumbral effects, the shaped-beam fluence map is convolved with a Gaussian kernel as described previously (Chetty et al 2000). There are several other approaches towards particle simulation in the treatment head for the purposes of treatment planning. A comprehensive review of these various implementations can be found in the recent paper by Verhaegen and Seuntjens (2003).

Monte Carlo (DPM) calculations were compared against those in the conventional UMPlan system, which uses the edge/octree algorithm (Fraass et al 1987). The edge/octree algorithm is based on a parameterization of the measured depth-dose curves and profiles at different depths in water using analytical models (Fraass et al 1987). The effect of tissue inhomogeneities is accounted for using an equivalent path length (EPL) correction, which performs a ray-trace from the source to the calculation point and scales the depth with the radiological density along that ray. The EPL correction applies only to primary photons; lateral electron transport and the distribution of scattered photons are ignored. The octree/edge algorithm with EPL inhomogeneity-correction algorithm as implemented in UMPlan will be referred to as the EPL algorithm throughout the rest of this paper.

\subsection{Beam model adjustments and phantom geometries}

A phantom, illustrated in figure 1, was simulated for the purposes of dose calculations in this study. The simulated phantom, consisting of uniform density water equivalent $\left(\rho=1.0 \mathrm{~g} \mathrm{~cm}^{-3}\right)$ and lung equivalent $\left(\rho=0.3 \mathrm{~g} \mathrm{~cm}^{-3}\right)$ materials with an embedded uniform density tumour $\left(\rho=1.0 \mathrm{~g} \mathrm{~cm}^{-3}\right)$, was designed with dimensions similar to those that one might encounter in an actual patient. For the purposes of beam fitting, single AP field calculations were initially performed in the phantom using the DPM and EPL algorithms. The phantom densities for 


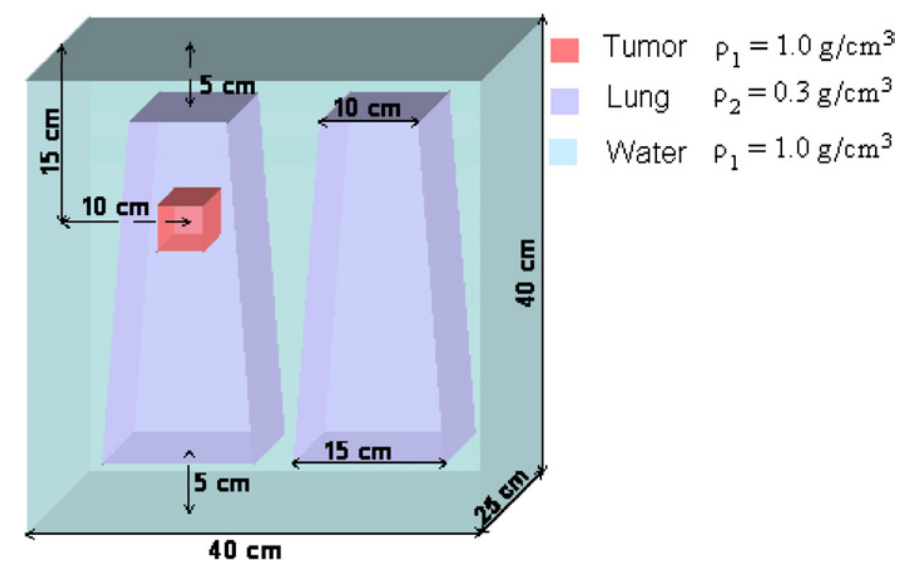

Figure 1. Phantom set-up for the treatment planning study. The tumour size was changed from $6 \mathrm{~cm}$ (side of cube) for the $6 \mathrm{MV}$ calculations to $2 \mathrm{~cm}$ for the $15 \mathrm{MV}$ treatment plans.

these AP field calculations were set to 1 -the intent here was to test the agreement between the beam models in the homogeneous situation. These calculations were subsequently analysed using 1D profiles and dose difference maps to quantify the differences in the beam models (see results, section 3.1). The EPL model parameters were then modified in the beam penumbral and low-dose regions to optimize the agreement with DPM. Adjustments were performed using $1 \mathrm{D}$ profiles at depths of $5,10,15$ and $20 \mathrm{~cm}$ in the homogeneous phantom, and the optimized fit represented the best trade-off in the agreement between DPM and EPL in the penumbral and low-dose regions at these various depths (see results, section 3.1).

Using these two different beam models, the initial fit (init_fit) and the optimized fit (best_fit) parameters, realistic treatment plans were designed for the phantom study. Beam arrangements consisted (in addition to the AP field) of PA and right lateral fields, along with two segmental fields (total of five beams) to produce a dose homogeneity of approximately $100 \pm 5 \%$ within the tumour in the homogeneous setting (with densities set to 1 ). Calculations were also performed in the heterogeneous phantom (using the actual tissue densities) for the init_fit and best_fit cases and compared with DPM. Additionally, calculations were carried out in phantoms with two different tumour sizes (cubes with sides 2 and $6 \mathrm{~cm}$ ) using 6 and $15 \mathrm{MV}$ photon beams, respectively. The field size for the $6 \mathrm{MV}, 6 \mathrm{~cm}$ tumour plan was $8 \times$ $8 \mathrm{~cm}^{2}$, while that for the $15 \mathrm{MV}, 2 \mathrm{~cm}$ tumour case was $4 \times 4 \mathrm{~cm}^{2}$. Finally, a patient treatment plan was included in the study to illustrate the influence of differences in the beam models on clinically realistic dose indices. As with the phantom study, calculations for the patient plan were performed in both the homogeneous (densities set to 1) and heterogeneous cases. It should be pointed out that, for all comparisons in this study, field shapes were defined by the MLC with the $x$ and $y$ jaw positions set to the most protruding leaf/leaves.

Transport parameters for the DPM calculations were as follows: the low-energy electron and photon cut-off values were 200 and $50 \mathrm{keV}$, respectively, and the step size was $2 \mathrm{~mm}$. DPM calculations were carried out with sufficient particles (of the order of 1 billion) to produce a statistical uncertainty $(1 \sigma)$ of less than $0.5 \%$ in the region of maximum dose. Dose was calculated in cubic voxels of sides $3 \mathrm{~mm}$ for both DPM and EPL algorithms. 


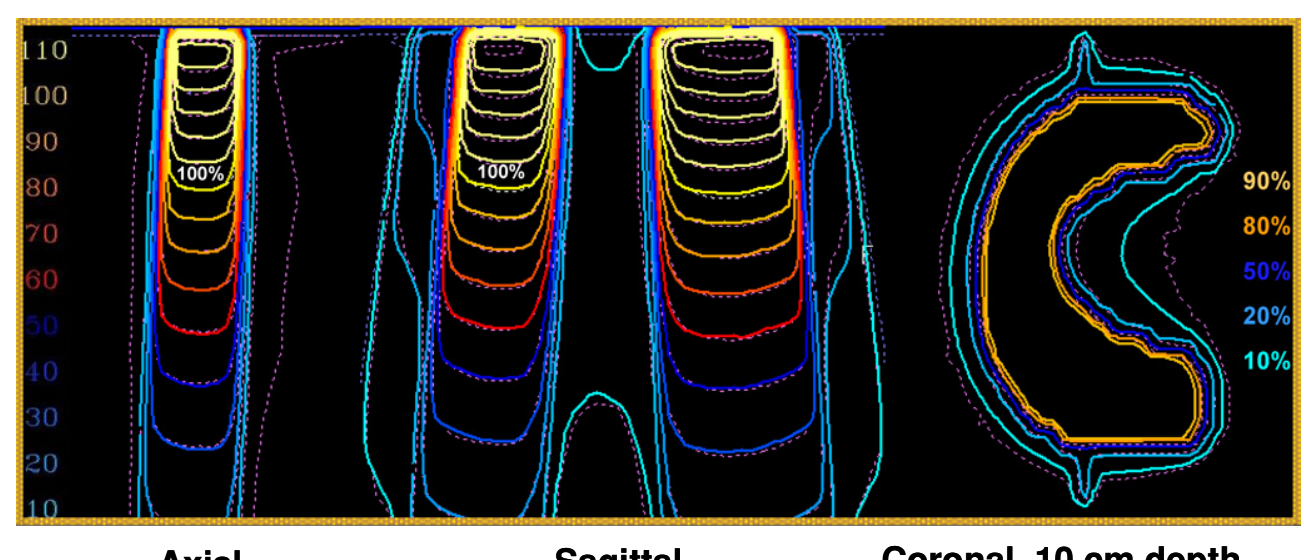

Axial

Sagittal

Coronal, $10 \mathrm{~cm}$ depth

Figure 2. Experimental verification of the MC source model used in the planning study (from Rosu et al (2003a)). The comparison shows ion chamber measurements (dashed lines) versus Monte Carlo (solid lines) for a 'C-shaped', MLC-defined field in the axial and sagittal planes. Measurements for the coronal comparison were obtained using film. The photon beam energy was $6 \mathrm{MV}$.

\subsection{Beam fit comparison metrics}

Differences in the dose distributions amongst the various EPL beam models (init_fit and best_fit) were compared against DPM using the following dose metrics: dose volume histograms (DVHs) for the tumour and normal lung tissue, mean lung dose (MLD), normal tissue complication probability (NTCP) for the normal lung tissue and dose in the EPL(init_fit and best_fit) plans to produce the same NTCP as in the DPM plan. MLD is the mean physical lung dose, calculated from the normal lung physical DVHs, as follows: MLD $=\sum{ }_{i} V_{i} \cdot D_{i}$, where $D_{i}$ is the physical dose in bin $i$, of volume $V_{i}$. NTCP was calculated using the LymanKutcher-Burman (LKB) model (Kutcher and Burman 1989) with fit parameters $(n=0.97$, $m=0.33, \mathrm{TD}_{50}=30.3 \mathrm{~Gy}$ ) initially derived from our previous lung dose escalation protocol (Hayman et al 2001), where doses were calculated using the edge/octree (EPL) algorithm. Normal lung volumes were calculated by subtracting the gross tumour volume (GTV) from the combined volume of both lungs. Isocentre doses were $84 \mathrm{~Gy}$ for the $6 \mathrm{MV}$ plan, $103.0 \mathrm{~Gy}$ for the $15 \mathrm{MV}$ plan and $66.0 \mathrm{~Gy}$ for the patient plan-doses were chosen on the basis of the volume of lung uniformly irradiated ( $\left.V_{\text {eff }}\right)$, much like in the protocol (Hayman et al 2001).

\section{Results and discussion}

\subsection{AP beam model comparisons}

For the purposes of verification of the Monte Carlo virtual source model used for simulating arbitrary field shapes, calculations were compared with measurements for a variety of MLCshaped fields. Figure 2 provides a comparison for a C-shaped field (6 MV photons) showing good agreement between measurement (dashed lines) and MC (solid lines) central axis (CAX) in the sagittal, axial and coronal views (Rosu et al 2003a). Other verification of the performance of the MC source model used in this study has been previously reported in studies by Rosu et al (2003a) and Chetty et al (2000, 2003).

Figure 3 illustrates CAX profiles in the $x$-direction (cross plane) for the $6 \mathrm{MV}$ AP beam $\left(8 \times 8 \mathrm{~cm}^{2}\right)$ at depths of (a) $5 \mathrm{~cm}$ and (b) $10 \mathrm{~cm}$. Note that these calculations were performed 

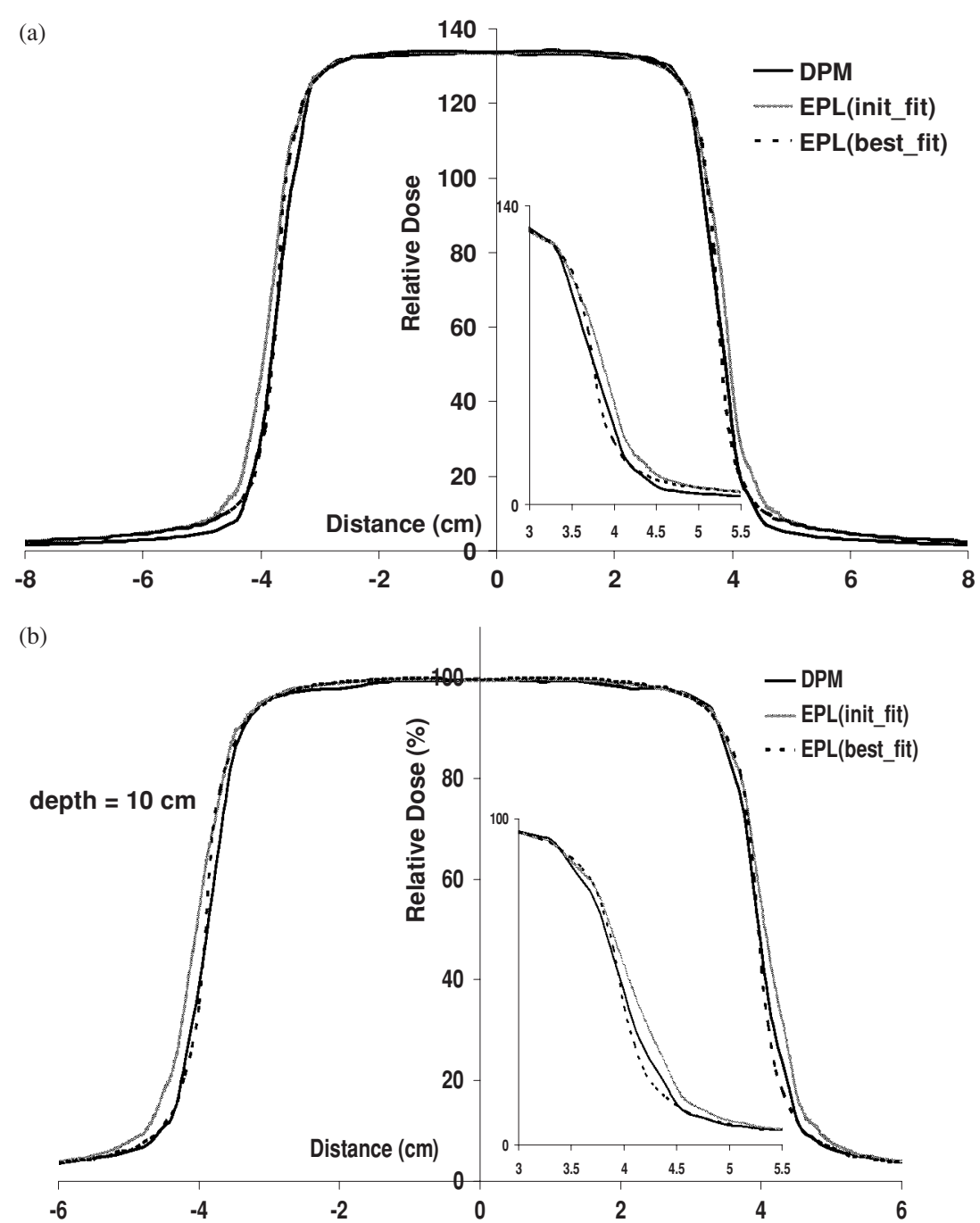

Figure 3. Central $x$-axis profiles for the $6 \mathrm{MV}\left(8 \times 8 \mathrm{~cm}^{2}\right)$, AP beam in the homogeneous $(\rho=1)$ phantom, at depths of (a) $5 \mathrm{~cm}$ and (b) $10 \mathrm{~cm}$. Plots are shown for the DPM, EPL(init_fit) and EPL(best_fit) calculations. The curves were normalized to a depth of $10 \mathrm{~cm}$ along the central axis.

in the homogeneous case. Curves were normalized at a depth of $10 \mathrm{~cm}$ along the CAX. Magnified views of the profile penumbral regions are presented in the inset figures. The improvement in the EPL beam fit (relative to DPM) using the optimized fit parameters (best_fit) versus the initial parameters (init_fit) is evident in the penumbral regions. The high- and low-dose regions (doses greater than $90 \%$ and less than $10 \%$, respectively) were relatively unchanged. While the init_fit consistently overestimates the penumbral dose, the best_fit provides a better tradeoff, and although the best_fit profiles show a somewhat sharper gradient, the overall agreement is better. The largest discrepancy in the init_fit profiles (relative to DPM) is $1.5 \mathrm{~mm}$ in the penumbral region (dose level of $\sim 25 \%$ at $10 \mathrm{~cm}$ depth), while that for the best_fit profiles is within $1 \mathrm{~mm}$ and on average within $0.5 \mathrm{~mm}$. Similar results were noted for the profiles at depths of 15 and $20 \mathrm{~cm}$ as well as for profiles along the $y$-axis. 

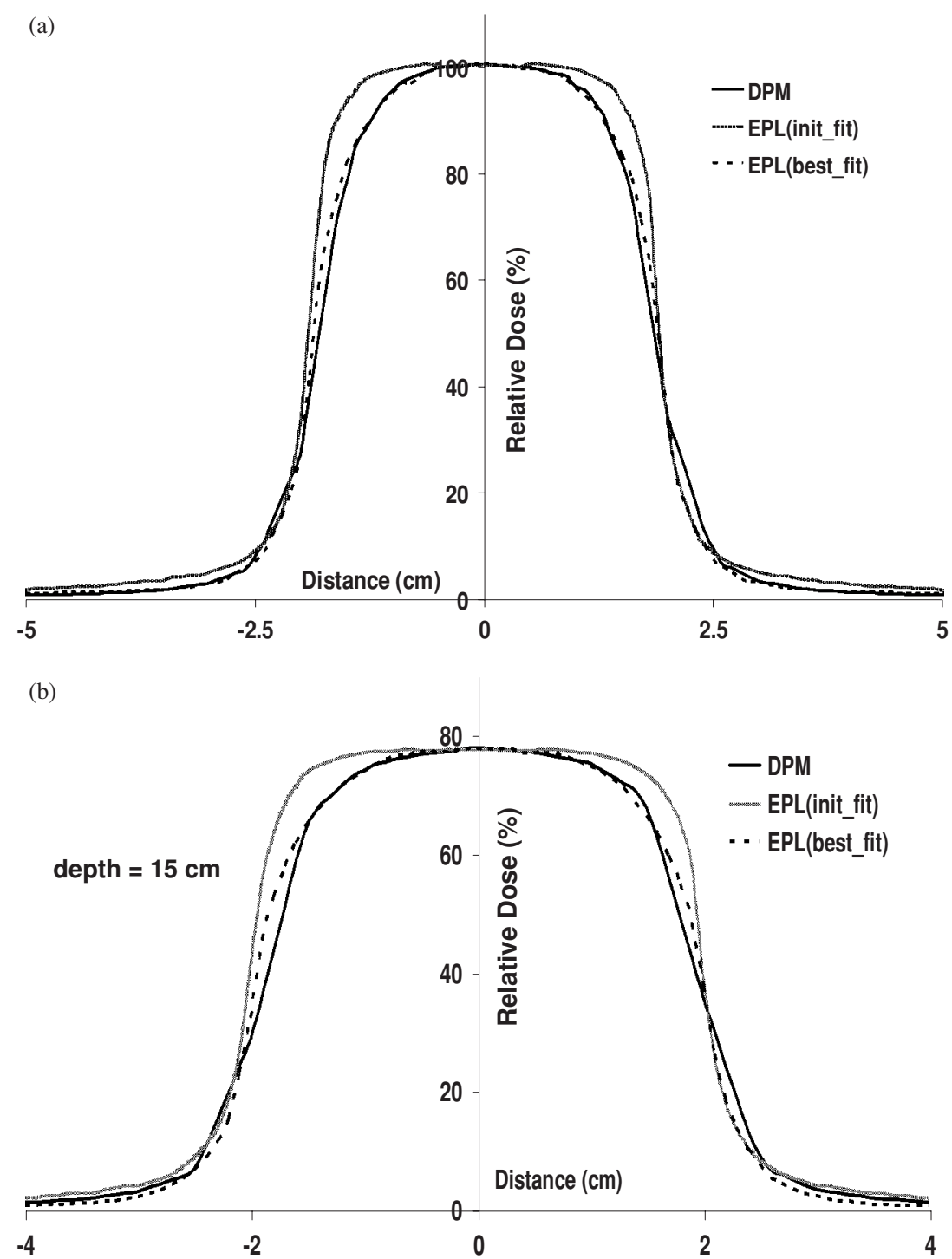

Figure 4. Central $x$-axis profiles for the $15 \mathrm{MV}\left(4 \times 4 \mathrm{~cm}^{2}\right)$, AP beam in the homogeneous $(\rho=1)$ phantom, at depths of (a) $10 \mathrm{~cm}$ and (b) $15 \mathrm{~cm}$. Plots are shown for the DPM, EPL(init_fit) and EPL(best_fit) calculations. The curves were normalized to a depth of $10 \mathrm{~cm}$ along the central axis.

A similar analysis for the AP beam fitting procedure (in the homogeneous phantom) was performed for the $15 \mathrm{MV}$. The beam field size was $4 \times 4 \mathrm{~cm}^{2}$. Figure 4 shows 1D CAX profiles along the $x$-axis at depths of (a) $10 \mathrm{~cm}$ and (b) $15 \mathrm{~cm}$. Curves were normalized at a depth of $10 \mathrm{~cm}$ along the CAX. The EPL best_fit provides a markedly improved fit over the init_fit relative to the DPM profiles. Due to the relatively small field size $\left(4 \times 4 \mathrm{~cm}^{2}\right)$, modification of the EPL model penumbral parameters also influenced the high-dose shoulder region of the profile; the largest discrepancies in the init_fit profiles (relative to DPM) are 7.4\% at the profile shoulders (dose region between $85 \%$ and $95 \%$ ) and $2.6 \mathrm{~mm}$ in the penumbral region. Agreement for the best_fit profiles in the penumbra is within $1 \mathrm{~mm}$ and within $2 \%$ 


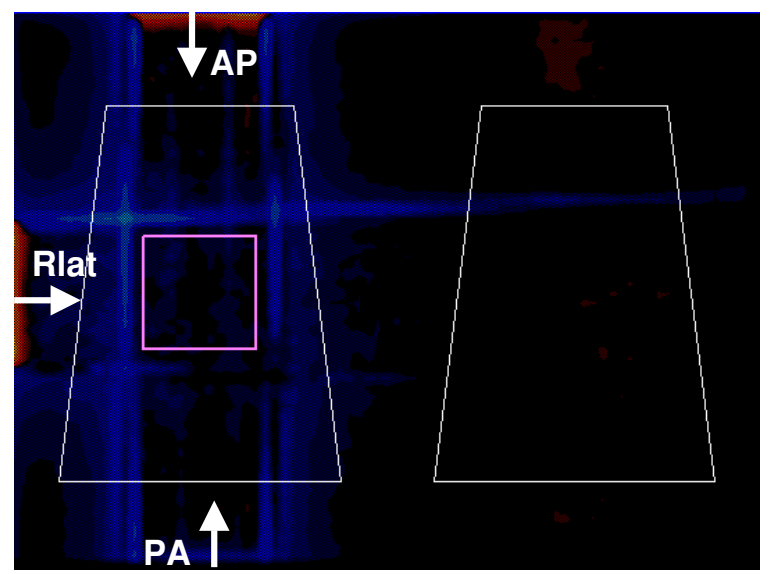

(a)

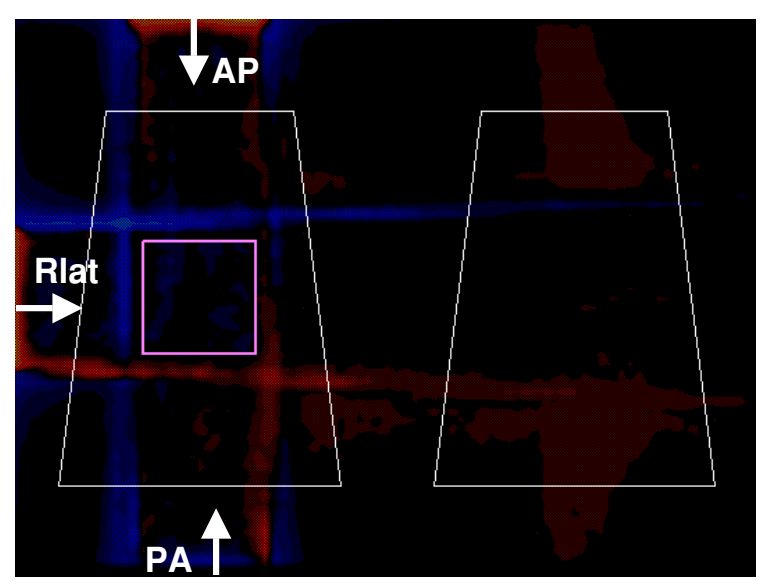

(b)

Figure 5. Colour wash dose difference maps for the $6 \mathrm{MV}$, five-field treatment plan in the homogeneous $(\rho=1)$ case shown for: (a) DPM-EPL(init_fit) and (b) DPM-EPL(best_fit). The dark (blue) regions along the beam edges represent negative differences. Maximum differences are $-25 \%$ (on average within $2 \mathrm{~mm}$ distance-to-agreement) along the beam edge in (a), and $-10 \%$ (on average within $0.5 \mathrm{~mm}$ distance-to-agreement) along the penumbra in (b). Excluding the build-up region (not included in the beam fitting), the light (red) regions in (b) show positive differences, which were all less than $3 \%$. Regions in black are within $\pm 1 \%$ agreement.

in the high-dose region above $90 \%$. Similar results were found for the profiles at depths of 5 and $20 \mathrm{~cm}$ as well as for profiles along the $y$-axis. Dose difference maps (DPM-EPL) for $15 \mathrm{MV}$ (though not presented here) showed notably improved agreement (relative to DPM) in the profile and penumbral and shoulder regions when calculations were performed with the EPL best_fit versus the init_fit.

\subsection{Phantom planning calculations}

The EPL beam parameters for the initial fit (init_fit) and the optimized fit (best_fit) determined in the above analysis for AP beams were applied to a multiple-beam (five-field) treatment plan. Illustrated in figure 5 are dose difference maps for the $6 \mathrm{MV}$ plan in the homogeneous case 


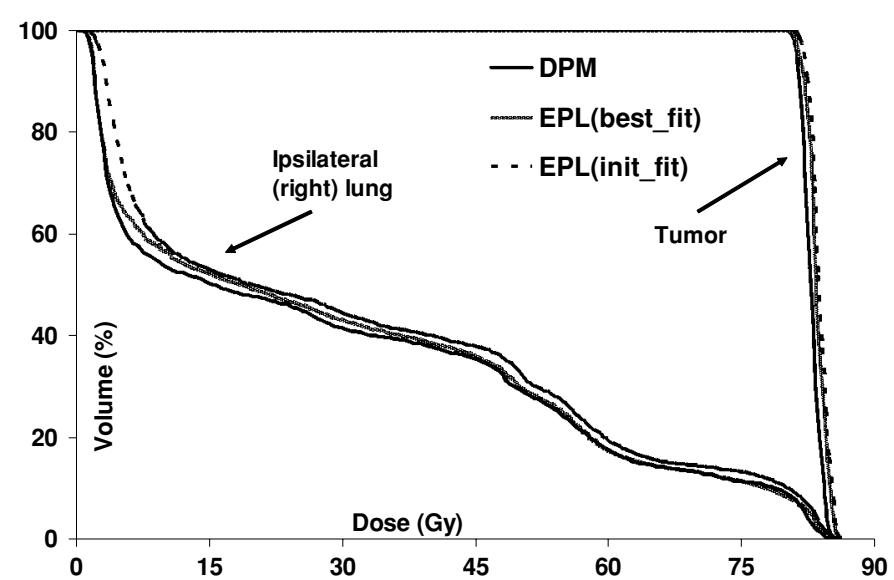

(a)

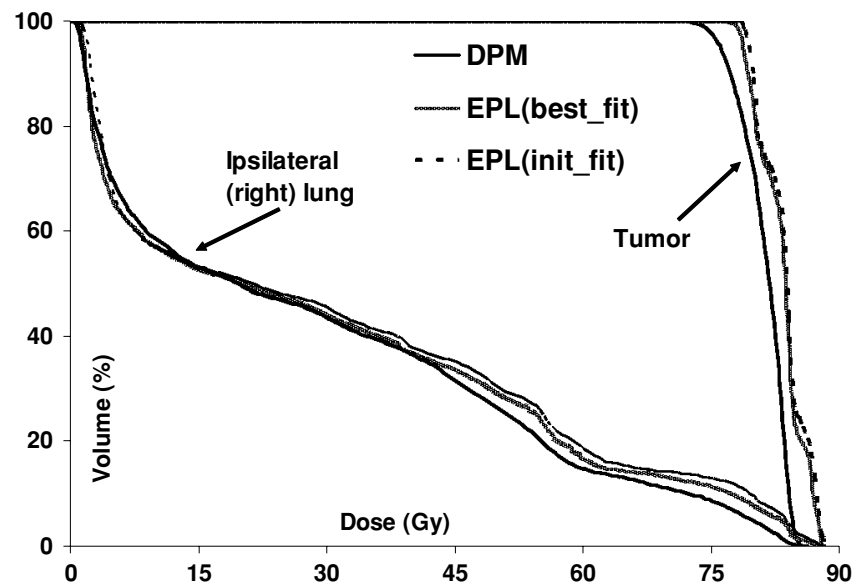

(b)

Figure 6. DVHs for the tumour and ipsilateral (right) lung and for the $6 \mathrm{MV}$, five-field treatment plan in (a) the homogeneous $(\rho=1)$ phantom and (b) the heterogeneous phantom. DVHs are included for the DPM, EPL(init_fit) and EPL(best_fit) calculations.

for (a) DPM-EPL(init_fit) and (b) DPM-EPL(best_fit). There is improved overall agreement with the best_fit parameters relative to the init_fit, particularly in the penumbral region, where the negative dose differences (cold spots) are substantially reduced. DVHs for the ipsilateral (right) lung and the tumour are shown in figures 6(a) and (b) for the homogeneous (densities set to 1) and heterogeneous phantom, $6 \mathrm{MV}$, treatment plans respectively. Note that a tumour with side $6 \mathrm{~cm}$, and field sizes of $8 \times 8 \mathrm{~cm}^{2}$ were used in this example. The prescribed dose was 84 Gy to the isocentre. The homogeneous DVHs (figure 6(a)) show that while the tumour is relatively unaffected by the difference in beam models, the dose to the ipsilateral (right lung) is influenced by these differences; the EPL(best_fit) DVH is in better agreement with that of DPM and more so in the region less than $15 \mathrm{~Gy}$. Values for the MLD, NTCP and dose to restore the NTCP for the DPM calculation (prescribed to $84 \mathrm{~Gy}$ at the isocentre) are presented for the homogeneous, $6 \mathrm{MV}$ plan, in table 1(a). MLD and \% NTCP calculated with EPL(best_fit) are in good agreement with the respective DPM values-this is also reflected in the dose 
Table 1. 6 MV treatment plan dose indices: mean lung dose (MLD), normal tissue complication probability (\%NTCP) and the dose to restore the DPM NTCP, for (a) homogeneous phantom and (b) heterogeneous phantom. The prescribed dose to the isocentre was 84 Gy. NTCP is shown for the normal lung tissue defined as the sum of the left and right lung volumes excluding the GTV.

\begin{tabular}{llll}
\hline & $\begin{array}{l}\text { MLD } \\
(\mathrm{Gy})\end{array}$ & $\begin{array}{l}\text { NTCP } \\
(\%)\end{array}$ & $\begin{array}{l}\text { Dose to restore } \\
\text { DPM NTCP }(\mathrm{Gy})\end{array}$ \\
\hline \multicolumn{5}{c}{ (a) Homogeneous phantom } \\
DPM & 14.64 & 6.32 \\
EPL(best_fit) & 14.84 & 6.60 & 82.77 \\
EPL(init_fit) & 15.84 & 7.96 & 77.74 \\
\multicolumn{5}{c}{ (b) Heterogeneous phantom } \\
DPM & 15.38 & 7.23 & \\
EPL(best_fit) & 15.70 & 7.74 & 82.12 \\
EPL(init_fit) & 16.38 & 8.78 & 78.76 \\
\hline
\end{tabular}

to restore the DPM NTCP, which is approximately 83 Gy for EPL(best_fit). On the other hand, larger differences are found for the EPL(init_fit) values; the dose to restore the DPM NTCP is now $78 \mathrm{~Gy}$, which represents a 6 Gy difference from the DPM prescribed dose. As this represents a dose change of more than one fraction (for a typical fraction size of $2 \mathrm{~Gy}$ ), we can consider this a clinically significant difference (Ten Haken et al 1997, Lujan et al 1999, Rosu et al 2003b). The fact that substantial differences are found in the lung doses in the homogeneous phantom suggests that differences of the order of $2 \mathrm{~mm}$ in the penumbral beam model can significantly impact doses to structures outside the target - these differences must be considered when comparing the algorithms in the heterogeneous phantoms.

Figure 6(b) shows DVHs for the $6 \mathrm{MV}$ treatment plan in the heterogeneous setting (using the actual physical densities). For the tumour, the DPM DVH shows a dose underestimation relative to the EPL DVHs, a well-known effect due to the lateral transport of electrons from the tumour into the lower density lung. For the lung, it is evident that the DPM DVH shows an increased volume receiving smaller doses (less than $15 \mathrm{~Gy}$ ) and a reduced volume receiving higher doses (greater than $45 \mathrm{~Gy}$ ) relative to the homogeneous treatment plan. This is consistent with the 'smearing' of dose into the lung as a result of lateral electron transport, which is correctly accounted for with Monte Carlo. DVHs for the EPL algorithm, on the other hand, show little change versus the homogeneous plan, as electron transport is not accounted for. The dose to restore the DPM NTCP in the heterogeneous plan (table 1(b)) is 82 Gy with EPL(best_fit) and 79 Gy with EPL(init_fit). A possible reason for this relatively small change in the EPL(best_fit) dose is that the lateral electron transport effect is small given the relatively large field size $\left(8 \times 8 \mathrm{~cm}^{2}\right)$ and the lower photon energy $(6 \mathrm{MV})$. Note also that a large fraction of the difference in the heterogeneous phantom for the EPL(init_fit) calculation is a result of the difference in the beam model in the homogeneous phantom; had the EPL(init_fit) been better modelled in the homogeneous plan, the differences in the inhomogeneous case may well have been smaller in this particular example, as in the case with EPL(best_fit). This illustrates that accurate beam fitting and differences in the beam model are important considerations in the comparison of dose algorithms in heterogeneous tissues.

Figures 7(a) and (b) show DVHs for the $15 \mathrm{MV}$ treatment plans in the homogeneous and heterogeneous phantoms, respectively. Curves are plotted for the ipsilateral (right) lung and tumour. For the $15 \mathrm{MV}$ example, a tumour of side $2 \mathrm{~cm}$ was chosen and the field size was $4 \times$ $4 \mathrm{~cm}^{2}$. The prescribed dose to the isocentre was $103 \mathrm{~Gy}$. In the homogeneous case (figure 7(a)), the EPL(best_fit) lung DVH is in better agreement with that of DPM versus the EPL(init_fit) 


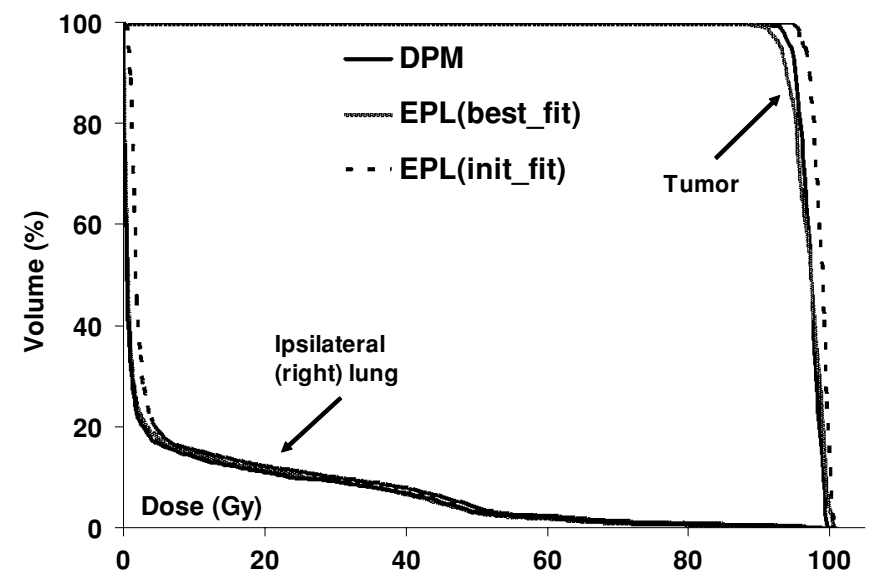

(a)

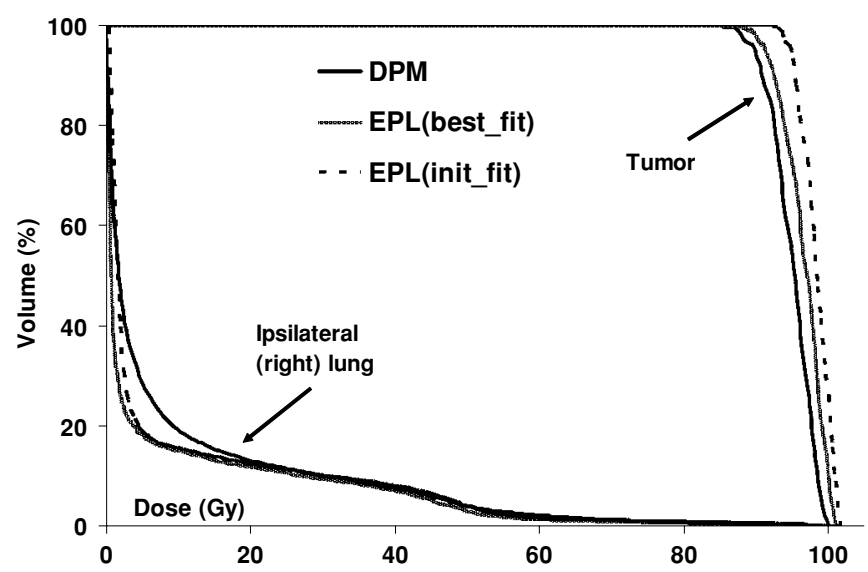

(b)

Figure 7. DVHs for the tumour and ipsilateral (right) lung and for the $15 \mathrm{MV}$, five-field treatment plan in (a) the homogeneous $(\rho=1)$ phantom and (b) the heterogeneous phantom. DVHs are included for the DPM, EPL(init_fit) and EPL(best_fit) calculations.

lung DVH - a similar trend was noted for the $6 \mathrm{MV}$ plan. For the tumour, the DVH differences between EPL(init_fit) and DPM are relatively large; this is because the adjustment of the EPL model penumbral parameters also influenced the high-dose shoulder region of the profile owing to the small field size used in this plan (see section 3.1). Dose metrics for the homogeneous, 15 MV plan are shown in table 2(a). Values for the MLD and \%NTCP are in relatively good agreement between DPM and EPL(best_fit) with larger differences found for EPL(init_fit). The dose to restore the DPM NTCP was 102 Gy for the EPL(best_fit), which compares well with the prescribed isocentre dose of $103 \mathrm{~Gy}$. For the EPL(init_fit) calculation, the $0.57 \%$ NTCP is higher than that of DPM $(0.44 \%)$ implying that the treatment is more toxic to the lung in the case of EPL(init_fit). As a result, the dose to restore the DPM NTCP for the EPL(init_fit) calculation is lower ( $85 \mathrm{~Gy}$ ); it is significantly lower (by roughly 10 fractions) because, in addition to the very high prescription dose (103 Gy), the NTCP and $V_{\text {eff }}$ values for this particular case fall along the steepest region of the dose response curve. 
Table 2. 15 MV treatment plan dose indices: mean lung dose (MLD), normal tissue complication probability (\%NTCP) and the dose to restore the DPM NTCP, for (a) homogeneous phantom and (b) heterogeneous phantom. The prescribed dose to the isocentre was 103 Gy. NTCP is shown for the normal lung tissue defined as the sum of the left and right lung volumes excluding the GTV.

\begin{tabular}{lccc}
\hline & $\begin{array}{l}\text { MLD } \\
(\mathrm{Gy})\end{array}$ & $\begin{array}{l}\text { NTCP } \\
(\%)\end{array}$ & $\begin{array}{l}\text { Dose to restore } \\
\text { DPM NTCP (Gy) }\end{array}$ \\
\hline \multicolumn{4}{c}{ (a) Homogeneous phantom } \\
DPM & 3.87 & 0.44 & \\
EPL(best_fit) & 3.93 & 0.45 & 101.63 \\
EPL(init_fit) & 4.76 & 0.57 & 85.01 \\
\multicolumn{5}{c}{ (b) Heterogeneous phantom } \\
DPM & 5.54 & 0.71 & \\
EPL(best_fit) & 4.24 & 0.49 & 132.72 \\
EPL(init_fit) & 4.92 & 0.60 & 115.47 \\
\hline
\end{tabular}

DVHs for the $15 \mathrm{MV}$ heterogeneous plan are presented in figure 7(b). Here again, as in the case of $6 \mathrm{MV}$, we find a significant reduction in the tumour DVHs with DPM relative to EPL due to the lateral electron transport into the lung. Note that this reduction is more pronounced for EPL(init_fit) since the init_fit model parameters were not optimally fit in the homogeneous case (see figure 7(a)). The dose to the normal lung tissue (right lung) changes substantially with DPM in the $15 \mathrm{MV}$ heterogeneous plan. The DPM DVH shows a considerable increase in the volume receiving a dose less than $20 \mathrm{~Gy}$ relative to the homogeneous plan (figure 7(a)). This is due to the lateral electron transport effect which is exacerbated by the use of small field sizes $\left(4 \times 4 \mathrm{~cm}^{2}\right)$, a small tumour size $(2 \mathrm{~cm})$ embedded in low-density lung tissue, and high energy photons (15 MV). This effect results in an increase in the DPM NTCP and MLD (as seen in table 2(b)) relative to the homogeneous case; the NTCP, for example, increases from $0.44 \%$ (in the homogenous plan) to $0.71 \%$ (in the heterogeneous plan), causing the DPM dose to the lung to be more toxic versus the EPL calculations. Note also in table 2(b) that neither the NTCP nor the MLD shows much change with either EPL init_fit or best_fit models in the heterogeneous case (relative to the homogeneous plan) because the EPL algorithm does not correctly account for lateral electron transport in the lung. As a consequence of the increased toxicity with the DPM doses to lung, the dose to restore the DPM NTCP for the EPL calculations is increased - the magnitude of the large increase (table 2(b)) is in part due to the high prescription dose and the steepness of the dose-response curve at which these doses are calculated. We also see for the EPL(best_fit) model that the dose to restore the DPM NTCP, although in good agreement with the prescribed dose in the homogeneous plan (table 2(a)), is considerably different ( $\sim 30 \%$ higher than the prescribed dose) in the heterogeneous plan (table 2(b)), unlike the case for the $6 \mathrm{MV}$ plan (see table 1). This suggests that, in situations where lateral electron transport effects are emphasized (such as in the $15 \mathrm{MV}$ example), inhomogeneity effects may be more significant than the beam model differences found in the homogeneous setting.

\subsection{Patient treatment plan}

Figure 8 shows dose difference maps (DPM-EPL), for example a lung patient treatment plan consisting of three, conformal, $6 \mathrm{MV}$ photon beams. The dose difference maps were generated from treatment plans in the homogeneous case (i.e., with patient densities set to 1) and are shown (in the central coronal plane) specifically for (a) DPM-EPL(init_fit) and 


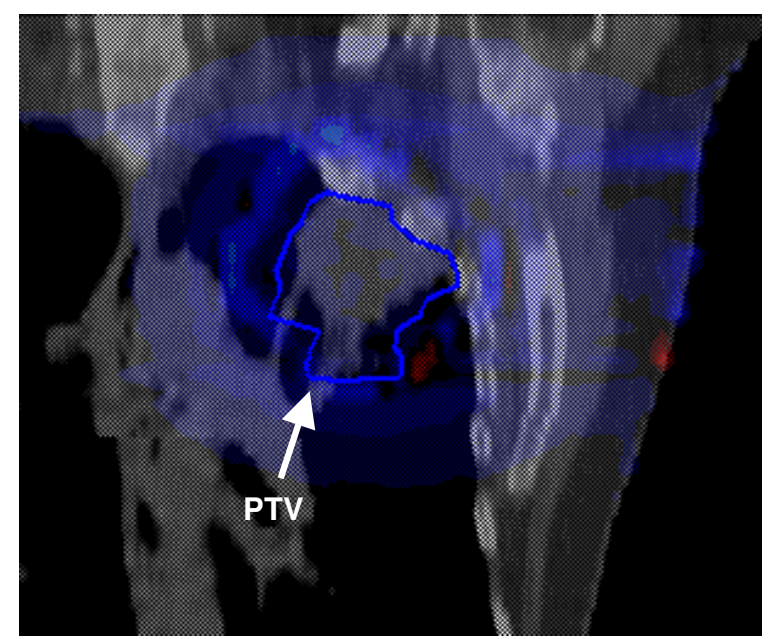

(a)

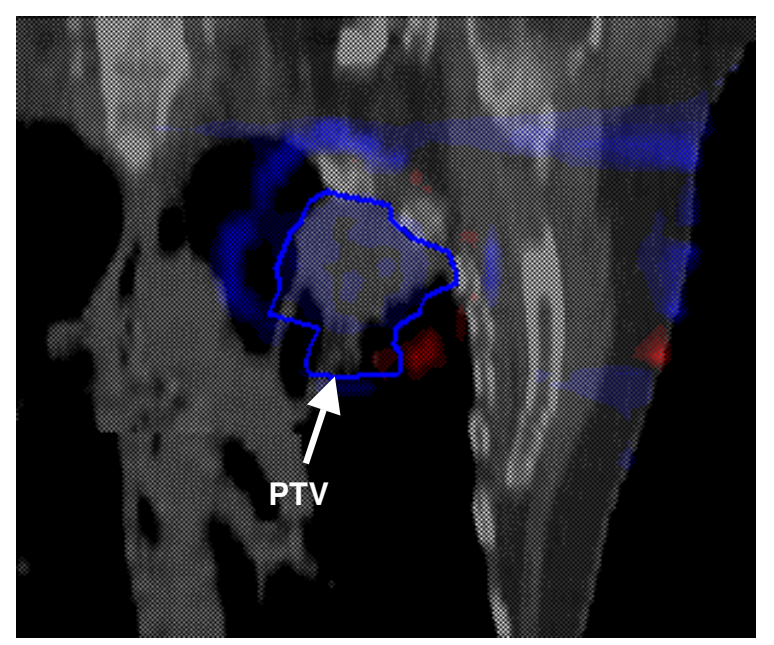

(b)

Figure 8. Colour wash dose difference maps for the conformal three-field, $6 \mathrm{MV}$ treatment plan in the homogeneous $(\rho=1)$ case shown for (a) DPM-EPL(init_fit) and (b) DPM-EPL(best_fit). The dark (blue) regions represent negative differences and range up to $-15 \%$ (on average less than $-7 \%$ ) in (a) and up to $-10 \%$ (on average less than $-3 \%$ ) in (b). Regions shown in light (red) indicate positive differences, which are all less than $3 \%$ (excluding the surface). Regions in black are within $\pm 1 \%$ agreement.

(b) DPM-EPL(best_fit). Results for this patient treatment plan were very similar to those for the $6 \mathrm{MV}$ phantom study described previously. The EPL(best_fit) shows better overall agreement when compared with DPM; average agreement is well within $\pm 3 \%$ (figure $8(\mathrm{~b})$ ), whereas larger negative differences $(-10 \%$ to $-15 \%)$ are found in the EPL(init_fit) difference map (figure 8(a)). DVHs for the normal lung tissue and tumour in the homogeneous and heterogeneous treatment plans, although not shown here, also followed similar trends to those for the $6 \mathrm{MV}$ phantom study described in section 3.2. To summarize, in the homogeneous treatment plan, the target DVHs calculated with DPM, EPL(best_fit) and EPL(init_fit) were all 
Table 3. Conformal, 6 MV patient treatment plan dose indices: mean lung dose (MLD), normal tissue complication probability (\% NTCP) and the dose to restore the DPM NTCP, for (a) homogeneous plan and (b) heterogeneous plan. The prescribed dose to the isocentre was 66 Gy. NTCP is shown for the normal lung tissue defined as the sum of the left and right lung volumes excluding the GTV.

\begin{tabular}{lcll}
\hline & $\begin{array}{l}\text { MLD } \\
(\text { Gy })\end{array}$ & $\begin{array}{l}\text { NTCP } \\
(\%)\end{array}$ & $\begin{array}{l}\text { Dose to restore } \\
\text { DPM NTCP (Gy) }\end{array}$ \\
\hline \multicolumn{4}{c}{ (a) Homogeneous plan } \\
DPM & 5.22 & 0.65 \\
EPL(best_fit $)$ & 5.16 & 0.64 & 66.59 \\
EPL(init_fit $)$ & 5.51 & 0.71 & 62.53 \\
& (b) Heterogeneous plan \\
DPM & 5.75 & 0.75 \\
EPL(best_fit $)$ & 5.87 & 0.79 & 64.38 \\
EPL(init_fit) & 6.13 & 0.84 & 61.82 \\
\hline
\end{tabular}

in good agreement. For the normal tissue, as expected, the EPL(best_fit) DVH was in better agreement with DPM versus the EPL(init_fit) DVH. In the heterogeneous treatment plan, DPM predicted a lower dose to the tumour relative to EPL, and for the normal lung tissue showed that the percentage volume receiving lower doses is increased while that receiving higher doses is reduced compared with the homogeneous plan. Dose indices for the homogeneous and heterogeneous patient plans are shown in tables 3(a) and (b), respectively. The MLD and NTCP calculated with EPL(best_fit) are in relatively good agreement with DPM in the homogeneous case (table 3(b)); larger differences are observed with the EPL(init_fit) calculations. The dose to restore the DPM NTCP in the homogeneous case was 66.6 Gy

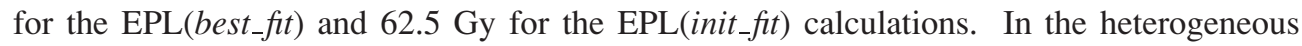
plan, the dose to restore the DPM NTCP is reduced by close to one fraction for the EPL(best_fit) suggesting that the inhomogeneity differences between DPM and EPL may be clinically significant in this example.

\section{Conclusion}

The results of this study indicate that consideration of differences in the beam model is important in comparing dose calculation algorithms in lung cancer treatment planning. Differences of the order of $2 \mathrm{~mm}$ in the profile penumbral regions in the homogeneous setting can contribute to the overall differences noted in the heterogeneous patient tissues, leading to inaccurate dose estimation to these tissues. Even though the magnitude of beam model differences will be dependent on several factors, such as the beam energy and orientation, field size, location and size of tumour, etc, we argue that no matter how small, these differences must be assessed and accounted for in order to perform an unbiased comparison between dose algorithms in the heterogeneous setting. As MC moves closer to the clinic, the question of how MC will impact patient outcome becomes increasingly important. Without first establishing accurate correlation between MC doses and those calculated with conventional algorithms, such as the EPL model (which have been previously tied to patient outcome), it will be very difficult to elicit the clinical benefit of MC. 


\section{Acknowledgments}

This work has been supported in part by NIH Grant P01-CA59827. The authors would like to thank Neelam Tyagi for her assistance with development of the Monte Carlo virtual source model.

\section{References}

Arnfield M R et al 2000 The impact of electron transport on the accuracy of computed dose Med. Phys. 27 1266-74 Chetty I J et al 2003 Photon beam relative dose validation of the DPM Monte Carlo code in lung-equivalent media Med. Phys. 30 563-73

Chetty I J, DeMarco J J and Solberg T D 2000 A virtual source model for Monte Carlo modeling of arbitrary intensity distributions Med. Phys. 27 66-172

Chetty I J et al 2004 Accounting for center-of-mass target motion using convolution methods in Monte Carlo-based dose calculations of the lung Med. Phys. 31 925-32

DeMarco J J, Solberg T D and Smathers J B 1998 A CT-based Monte Carlo dosimetry tool for radiotherapy treatment planning and analysis Med.Phys. 25 1-11

Deng J, Jiang S B, Li J, Pawlicki T and Ma C M 2000 Photon beam characterization and modeling for Monte Carlo treatment planning Phys. Med. Biol. 45 411-27

Fix M K et al 2001 A multiple source model for 6 MV photon beam dose calculations Phys. Med. Biol. 46 1407-28

Fraass B A et al 1987 3-D treatment planning: V. A fast 3-D photon calculation model The Use of Computers in Radiation Therapy ed I A D Bruinvis et al (Amsterdam: Elsevier/North-Holland) pp 521-5

Hayman J A et al 2001 Dose escalation in non-small cell lung cancer using 3-dimensional conformal radiation therapy: update of a phase I trial J. Clin. Oncol. 19 127-36

Heath E and Seuntjens J 2003 Development and validation of a BEAMnrc component module for accurate Monte Carlo modelling of the varian dynamic millennium multileaf collimator Phys. Med. Biol. 24 4045-63

Kutcher G J and Burman C 1989 Calculation of complication probability factors for nonuniform normal tissue irradiation: the effective volume method Int. J. Radiat. Oncol. Biol. Phys. 16 1623-30

Lujan A E, Larsen E W, Balter J M and Ten Haken R K 1999 A method for incorporating organ motion due to breathing into 3D dose calculations Med. Phys. 26 715-20

Rogers D W O et al 1995 BEAM: a Monte Carlo code to simulate radiotherapy treatment units Med. Phys. 22 503-24

Rosu M et al 2003a Experimental validation of a virtual source model for MLC-shaped dose calculations using the dose planning method (DPM) Monte Carlo code Med. Phys. 301515 (abstract)

Rosu M et al 2003b Alterations in normal liver doses due to patient motion Int. J. Radiat. Oncol. Biol. Phys. 57 1472-9

Schach von Wittenau A E et al 2000 Correlated histogram representation of Monte Carlo derived medical accelerator photon output phase space Med. Phys. 26 1196-212

Sempau J, Wilderman S J and Bielajew A F 2000 DPM, a fast, accurate Monte Carlo code optimized for photon and electron radiotherapy treatment planning dose calculations Phys. Med. Biol. 45 2263-91

Ten Haken R K et al 1997 Potential benefits of eliminating planning target volume expansions for patient breathing in the treatment of liver tumors Int. J. Radiat. Oncol. Biol. Phys. 38 613-17

Verhaegen F and Seuntjens J 2003 Monte Carlo modeling of external radiotherapy photon beams Phys. Med. Biol. 48 107-64 